\title{
Leutnant Gustl und seine Ehre
}

\section{Gero von Wilpert}

Schnitzlers Leutnant Gustl stünde, wenn er noch lebte - und Gegenteiliges ist dem Amte nicht bekannt - jetzt etwa in seinem 110. Lebensjahr: ein stattliches Alter, wenn man das Wort 'würdig' vermeiden will, und ebenso stattlich ist der Berg an Sekundärliteratur, die die Novelle seit ihrem Erstdruck in der Neuen Freien Presse vom 25. Dezember 1900 hervorgerufen hat. Allein im letzten Vierteljahrhundert hat sie fast fünfundzwanzig Einzelinterpretationen ${ }^{1}$ gefunden, und das ist mit schöner RegelmäBigkeit eine pro Jahr.

Das darf indessen nicht zu der Annahme verführen, wir verstünden die Novelle. heute besser und tiefer als die Zeitgenossen um die Jahrhundertwende und ihre Probleme hätten heute weitgehend eine Lösung gefunden. Es gibt im Gegenteil begründeten Anlaß zu der Vermutung, daß wir uns zusehends weiter von einem echten Verständnis entfernen.

Gewiß sind viele ihrer Aspekte heute deutlicher ins Bewußtsein gehoben worden. Der geniale Kunstgriff, die ganze Erzählung als inneren Monolog der Hauptfigur zu gestalten, ist zur Genüge analysiert und gewürdigt worden. Die soziale Problematik, die Diskrepanz von sozialem Anspruch und sozialer Wirklichkeit des k. u. k. Offizierskorps innerhalb einer in Umwälzung begriffenen Welt, wird im Rückblick aus zeitlicher Distanz und auf zwischenzeitliche Entwicklungen sehr viel deutlicher. Und auch der Charakter des Leutnants - wenn man in diesem Zusammenhang von Charakter sprechen darf - ist dank der Hebammenkunst der Interpreten um viele Züge bereichert worden, deren einige durchaus sogar im Text selbst verankert sind.

Dagegen wissen wir immer noch nicht genau, aus welchen Kreisen Gustl stammt. Es gibt Interpreten, die ihn mit dem Grundton der Überzeugung für das Kleinbürgertum in Anspruch nehmen, ${ }^{2}$ andere, die ihn dem Beamtenstand zuschreiben, ${ }^{3}$ und schließlich jene, die ihre Hand dafür ins Feuer legen - und das sollten sie nicht tun - er stamme aus adliger Familie. ${ }^{4}$ Nicht, daß das ganz unwesentlich wäre für die Frage, ob Gustl sich an alte Familientraditionen klammert oder als Aufsteiger die Wertvorstellungen der Zjelklasse internalisiert. Wo uns heute nur diffuse Mutmaßungen möglich sind, muß für Schnitzler seine Aufsteigermentalität so eindeutig gewesen sein, daß er kein Wort darüber verliert. Und um aus der 'intentional fallacy' in eine 'argumentatio ex 
silentio' zu fallen: es wäre undenkbar, daß ein Leutnant Gustl adliger Herkunft einer solchen im Laufe seiner Krisennacht nicht gedacht hätte.

Als Ersatz für solche belanglosen Äußerlichkeiten hat das Tranchierbesteck der Psychoanalytiker uns reichlich mit Innenaufnahmen unseres Helden versehen. Was hat unser armer Leutnant nicht alles über sich ergehen lassen müssen!

Man hat ihn auf die Couch gelegt, man hat ihm einen Ödipus-Komplex insinuiert, 5 und zwar eine ganz private Sonderanfertigung Marke Oedipus substitutus, bei dem seine Schwester Klara den Mutter-Ersatz spiele ${ }^{6}$ und der Haß auf den Vater sich auf die beiden angeblichen Vaterfiguren des Doktors und des Bäckermeisters übertragen lasse. ${ }^{7} \mathrm{Ja}$, man hat, als der Bäckermeister seinen Säbel festhält und ihn zu zerbrechen droht, von einer auf das Vaterbild projizierten Kastrationsangst gesprochen, ${ }^{8}$ obwohl hier Objekt und Medium kurioserweise ineinanderfließen. Man hat ihm einen geheimen Todeswunsch untergejubelt, ${ }^{9}$ der zum Ausgang der Novelle in diametralem Gegensatz steht. Man hat ihm "homosexuelle Neigungen" bzw. eine "latente Homosexualität" zugeschrieben. ${ }^{10}$ Man hat ihm sogar eingeredet, die ganze Episode mit dem Bäckermeister habe sich gar nicht in der Wirklichkeit abgespielt, sondern sei eine bloße Ausgeburt seiner Phantasie gewesen, die sich fein säuberlich in Vaterhaß, Kastrationsangst und Todessehnsucht zerlegen lasse. ${ }^{11}$

Und man hat letzlich die Wurzel aller seiner Probleme in jener Nacht im Walde gefunden, in der der füntzehnjährige Gustl nach eigener Aussage (351) das einzige Mal Furcht gehabt hat, und es ist nur bedauerlich, daß die Einbildungskraft der Interpreten uns nur vage Andeutungen gibt, was in jener Nacht im Walde denn Furchterregendes vorgefallen sein mag. 12

Ich muß mir hier um der Ehre willen versagen, einen der psychologisch interessantesten Fälle der ganzen moderen Literatur näher darzustellen, von dem weder unser Held noch sein Erfinder auch nur die geringste Ahnung hatten. Denn dies alles, wäre es wahr, machte aus dem Wiener Leutnant Gustl eher einen Lieutenant Gustl aus Williamsburg, Tennessee.

Auch die Figuren-Ausschlachtung oder -Wiederverwertung durch die literarischen Kollegen hat nicht gefehlt - oder gerade "gefehlt": Leutnant Gustl wurde dramatisiert (durch Ernst Lothar, 1961), bühnenbearbeitet, verhörfunkt und ferngesendet, bis von Schnitzlers Figur wenig mehr als der Name blieb, ein charmanter Pralinésoldat, der erst von seiner Freundin - sie heißt jetzt Anna, gleicht aber genau der Christine und kommt direkt aus der Liebelei hereinspaziert - vom Tode des Bäckermeisters erfährt und als Anzahlung auf die Hochzeit ihrem Wunsch nachgibt, das Duell mit dem 
Doktor abzusagen. ${ }^{13}$ So einfach lösen sich Gustls Probleme achtzig Jahre später, durch simple Umkehrung von Anatols berühmtem Ausspruch "Ich bin heute entschieden nicht in der Stimmung zum Heiraten. Ich möchte absagen."14

Der stoffgeschichtlichen Forschung bleibt angesichts solcher Verballhornungen nur anzumerken, daß es sich hierbei nur um Nachahmungen in freier Anlehnung an Schnitzlers Figuren handelt, bei denen jede eventuelle Ähnlichkeit mit echten, lebenden literarischen Personen rein zufällig wäre. Darf man nun aber die freischöpferische Phantasie, wie zerstörerisch auch immer sie ist, die man den Literaten nachsieht, in gleichem Maße auch den Interpreten aus den Rängen der Literaturwissenschaft zugute halten?

Wer ist denn jener Leutnant Gustl, der sich deplaziert im Oratorium tödlich langweilt und nur sein Kulturbanausentum kaschiert, der nur wegen einer Freikarte, wegen Spielschulden und, weil seine Freundin ihn zugunsten eines zahlenden Liebhabers versetzt hat, dort gelandet ist und, um das Beste daraus zu machen, die Gelegenheit zu neuen Eroberungen nutzen will, der das einzige Buch, das er besitzt, noch nicht ausgelesen hat, und der keine Frau ansehen kann, ohne ans Bett, und keinen Mann, ohne an ein Duell zu denken?

Schnitzler selbst hat mit viel zu sanfter Ironie gesagt, Gustl sei "ein ganz netter, nur durch Standesvorurteile verwirrter Bursch [ . . .], der mit den Jahren gewiß ein tüchtiger und anständiger Offizier werden dürfte." 15 Oberflächliche Interpreten haben die Ironie übersehen, die Autorinterpretation aber für bare Münze genommen und sie nachgebetet:

Für Albert Soergel ${ }^{16}$ ist Gustl "ein guter Junge von nicht gerade großen Geistesgaben", für andere ${ }^{17}$ gar ein "harmlos-lebensfroher Leutnant", und die Zahl der Urteile von Gustl-Sympathisanten ließe sich beliebig vermehren. Ob AnatolReminiszenzen bei solcher Beurteilung eine Rolle gespielt haben, ob der Anflug von 'Weaner' Dialekt bereits als Charme verbucht wurde, wie das vor Herrn Karl so oft der Fall war, oder ob der durchgängige innere Monolog der Novelle vermittels Identifikation des Lesers Sympathien und damit positive Einstellung fördert, kann dahingestellt bleiben. Daß dagegen bei näherem Hinsehen von dem netten, feschen Operettenleutnant keine Spur übrigbleibt, wird sich noch erweisen.

Trotz der durchgehenden Verwendung des inneren Monologs ist Gustl gar nicht als Individuum, sondern als Typ dargestellt, und zwar in der für die Gesellschaft der Jahrhundertwende leitbildhaften Rolle des jungen Offiziers. Seine innere Hohlheit und 
seine geistige Unbedarttheit lassen ihn nur noch in dieser sozialen Funktion seinen persönlichen Wert sehen. So nimmt es wenig wunder, wenn er den militärischen Formalismus, den Phrasenschatz, die Verhaltensregeln und sozialen Konventionen seines Standes in einem Maße internalisiert hat, das alles Persönliche selbst aus seiner Rede- und Denkweise verdrängt und ihm zeitweise sogar den Selbstmord als eine Lust schmackhaft machen kann.

Da nicht menschlicher Wert und Individualität, sondern allein Würde und Ehre seines Standes seinen sozialen Status bestimmen, wird sein Dasein ein solches totaler Fassadenhaftigkeit, und er klammert sich unbewußt umso fester an diese Konventionen, als die sozialen Umwälzungen, vor allem das Aufkommen eines selbstbewußten Bürgertums, diesen Status und damit seine eigene Rolle in Frage zu stellen drohen. Entsprechend werden alle Gruppen, die seinen sozialen Status bedrohen oder anzweifeln, wie die Sozialisten, die Akademiker, die Reserveoffiziere und die Juden, zu Feindbildern erklärt, denen Gustl mit allen Mitteln seines Standes, insbesondere einer aus uneingestandenen Minderwertigkeitskomplexen genährten Aggression, entgegentritt.

Der junge Doktor, der ihn kürzlich auf einer Gesellschaft bewußt unpersönlich gefragt hat: "Sie werden mir doch zugeben, daß nicht alle thre Kameraden zum Militär gegangen sind, ausschließlich um das Vaterland zu verteidigen!" (341), mag damit gar keinen Affront, sondern nur eine Tatsachenfeststellung im Lichte der Vernunft gemeint haben. Gustl dagegen fühlt sich nicht nur persönlich herausgefordert, weil die Beobachtung den Tatsachen entspricht: Gustl konnte durch seinen Hinauswurf auf dem Gymnasium das geplante Ökonomiestudium nicht aufnehmen und wurde in die Kadettenanstalt gesteckt. Gerade weil der Wahrheitsgehalt der Feststellung, bei der Wahl des Offiziersberufs spielten neben idealistischen vor allem auch handfeste Interessen eine Rolle, unleugbar ist, sieht Gustl darin einen Angriff auf die Ehre des Militärs und dessen hohen Anspruch auf absoluten Idealismus, also auch auf seinen eigenen sozialen Status.

Er reagiert entsprechend mit einer Duellforderung, die sein feines Ehrgefühl auch den Vorgesetzten gegenüber dokumentiert und, da diese sein Verhalten gutheißen, also auch seiner Karriere förderlich sein wird: wieder dieselbe Verbindung von idealistischen und handfesten Interessen. Es tut dabei nichts zur Sache, daß Gustl mitunter den nötigen Zorn gar nicht mehr aufbringen kann (340), für die Ehre seines Standes und damit seine eigene ist jedes Mittel recht, und je größer der Wahrheitsgehalt der Anschuldigung, umso unerbittlicher die Zurückweisung mit allen martia- 
lischen Mitteln.

Während des Oratoriums jedoch beschäftigt das bevorstehende Duell Leutnant Gustl nur ganz am Rande. Da Duelle bei ihm bereits in Serie stehen, ist ihm um den Ausgang nicht bange. Im Vordergrund seines Interesses steht vielmehr die Frage nach der Tugendhaftigkeit oder nicht der weiblichen Anwesenden. Das für Gustl befremdliche kulturelle Milieu, in dem er sich nicht wohlfühlt, wird auf sein Niveau herabgezogen und dient nur zur Basis einer Heerschau für künttige Eroberungen. Auch dies und die Tatsache, daß Gustl den anderen männlichen Anwesenden ähnliche amusische Ambitionen unterstellt, ist typisch für das Standesbild, das Schnitzler hier entwirft.

Da Gustl auf der Kadettenanstalt das militärische Erfolgsgesetz Nr. 1 gelernt hat, nämlich zur rechten Zeit einsatzbereit am rechten Ort zu sein, da das demokratische Reihenprinzip des Schlangestehens bei der Verabfolgung der Garderobe aber gegen sein elitäres Bewußtsein verstößt, ${ }^{18}$ sucht er sich nach Ende des Konzerts durch rücksichtsloses Vordrängen ungerechtfertigte Vorteile zu verschaffen ("Machen Sie doch Platz", 343).

Als die Zivilisten seinem Übereifer Widerstand entgegensetzen und überdies die ins Auge gefaßte Eroberung bereits einen Begleiter gefunden hat, gerät er erstens in Erregung und zweitens in einen Wortwechsel mit dem vor inm stehenden Zivilisten, den er durch ein ebenso kurzes wie militärisches "Sie, halten Sie das Maul!"(343) abbrechen will.

Es wäre hier der Ort, zu fragen, auf welchen Voraussetzungen denn die Ehre des Offiziers eigentlich beruht. So gewiß die Bereitschaft, ihr Leben für "Volk und Vaterland" oder, was immer es sein mag, in die Bresche zu werfen, zu Ansehen und Würde der Kriegerkaste beitragen mag, können doch daraus noch keine gesellschaftlichen Vorrechte abgeleitet werden. Erst aus dem elitären Denken der Offizierskaste entfaltet sich ein Selbstbewußtsein, das aufgrund höherer Lebensart einen sozial höheren Rang und höhere gesellschaftliche Privilegien in Anspruch nehmen will. Solch elitäres Denken jedoch setzt zugleich ein höheres Menschentum voraus, das sich wennschon nicht biologisch-rassisch, dann in feinerem, vorbildhaftem Benehmen ("Conduite") und in höheren, nicht materiellen Werthaltungen der "öffentlichen Personen" dokumentieren kann.

So sicher es nun ist, daß für das Offizierskorps der k. u. k. Armee ein verfeinerter, vorbildlicher "Comment" auch im Umgang mit Zivilisten und Normalmenschen galt, die sie durch Vorbildlichkeit und Würde des Auftretens beeindrucken und gewinnen sollten, ebenso sicher ist es, daß Gustl mit seinem plebejischen "Sie, halten Sie das 
Maul!" gegen den guten Ton in allen Lebenslagen und gegen die Konvention der Vorbildhaftigkeit verstößt, daß er also die durch feinere Lebensart in Anspruch genommenen Privilegien durch pure Arroganz ersetzt und verrät.

Das kommt ihm auch sogleich zu Bewußtsein, wenn er im inneren Monolog reflektiert: "Das hätt' ich nicht sagen sollen, ich war zu grob." (343) Der innere Monolog, im Unausgesprochènen auch sonst nicht gerade von sprachlichem Zartgefühl getragen, bricht hier unversehens infolge eines Aggressionsstaus in die direkte Rede ein. Er führt damit zur direkten Konfrontation mit dem Bäckermeister Habetswallner, den Gustl zu seinem Erschrecken wiedererkennt.

Gustl, der die Gelegenheit zu einer Entschuldigung verstreichen läßt - und wann hätte der Typ Gustl sich je entschuldigt? - hat sich durch offiziersunwürdiges Benehmen, durch ein Vergreifen im Ton, ins Unrecht gesetzt. Menschlich ist der Bäckermeister durchaus gerechtfertigt, wenn er dem flegelhaften, arroganten Leutnant mit seinem deplazierten Schneid eine Lektion erteilen will. Er tut dies in aller Stille und ohne Aufsehen, wenn er den Griff von Gustls Säbel festhält, um ihn vor weiteren Ausschreitungen seines martialischen Imponiergehabes zu bewahren, und wenn er ihm unter vier Augen damit droht, den Säbel aus der Scheide zu ziehen, ihn öffentlich zu zerbrechen und die Stücke an sein Regimentskommando zu schicken. Er entzieht mit dieser Drohung Gustl die einzige Basis seiner sozialen Anmaßung und gibt ihm zu verstehen, daß es nach dem sprachlichen Ausrutscher gegen den Komment nur der Säbel ist, der Gustl einen sozialen Vorrang gibt, und daß auch dieser 'in seine Hand' gegeben ist. Mit der sicher nicht als Beleidigung, sondern als väterliche Zurechtweisung gedachten Verwarnung "Sie dummer Bub" (343) wird Gustl menschlich auf seinen Platz verwiesen. Und um der väterlichen Ermahnung zu dezenterem Benehmen auch nach außen hin jeden Anschein einer Konfrontation zu nehmen, scheiden sie mit einem "Habe die Ehre, Herr Leutnant, hat mich sehr gefreut - habe die Ehre." (344)

Es wird jedem Leser dieser Szene damals wie heute deutlich sein, auf wessen Seite Menschlichkeit, Ehrbarkeit und menschliche Würde stehen, und es liegt kein Grund vor, hier von "Verachtung für einen Träger dieser Ordnung" und von "unbekümmert brutalem Auftrumpfen" 19 besitzbürgerlichen Selbstbewußtseins zu reden. Gerade darin, daß der Bäckermeister nichts unternimmt, was dem auf Äußerlichkeiten bedachten Ansehen Gustls abträglich sein könnte, zeigen sich vielmehr menschliches Verständnis und menschliche Größe. Nicht so für Gustl, dessen Lebensnerv, dessen raison d'être, dessen Existenz mangels anderer menschlich-moralischer Qualitäten so sehr auf dem einen hypertrophen Begriff der Standesehre seiner Kaste als einer 
höherstehenden Lebensform beruht, daß inm eine objektive, sachliche Würdigung des Vorgefallenen gar nicht möglich ist.

Für unseren Zusammenhang ist es nun gleichgültig, ob man im Griff nach dem Säbel und der Drohung, inn zu zerbrechen, eine Kastrationsdrohung sehen will oder nicht. In dem Moment, wo der beschriebenen äußeren Handlung psychoanalytische Deutungen unterlegt werden, wird der Begriff der Ehre ohnehin obsolet. Gustl selbst, in dessen Weltbild Freud nie einen Stellenwert haben wird, macht sich darüber jedenfalls keine Gedanken. Für ihn ist der Griff nach seinem Säbel ein Griff nach seiner Ehre, und die Bezeichnung "Sie dummer Bub" erfüllt für ihn den Tatbestand der Beleidigung seiner Ehre.

Genau an diesem Punkt nun scheiden sich wiederum die Geister der Interpreten. Die einen, in den Werthaltungen des späten 20. Jahrhunderts befangen, denen Duell und Ehrenhandel vorsintfluttiche Atavismen sind, bedauern herablassend den dümmlichen Gustl, der nur "meint, in seiner Ehre gekränkt zu sein"20 oder sich "aufgrund einer schieren Nichtigkeit"21 zum Tode verurteilt. Sie beklagen entweder, daß ihm die Dickfelligkeit abgeht, die seine modernen Kollegen in dieser Situation an den Tag legen würden, oder daß ihm die Einsicht in die zeitbezogene Bedingtheit eines solchen Ehrenkodex mangelt, weil er zu ausschließlich konventionsgesteuert, von den Verhaltenserwartungen der Standesgenossen gelenkt sei. Wir können von solchen vernünftelnden Betrachtungen post festum in Zukunft absehen, weil ihre Deutung letztlich darauf hinausläuft, Schnitzler habe sich die Beschränktheit seines Helden zunutze gemacht, ihn in eine objektiv gar nicht gegebene existentielle Grenzsituation zu manövrieren, und verspotte also damit nur die Dummheit seines Helden. Trop de bruit pour une omelette!

Die Mehrzahl der Interpreten geht sehr zu Recht vorerst mit Gustls eigenem Befund der Situation aus der Sicht eines Offiziers der Jahrhundertwende überein, und ihr können wir einstweilen folgen.

Ihmzufolge erfüllen der Griff nach dem Säbel, die Drohung der Ehrabschneidung und das "Sie dummer Bub" unabhängig davon, ob sie so gemeint waren oder nicht, objektiv den Tatbestand der Ehrenkränkung.

Die konventionell richtige, vorschriftsmäßige Lösung des Konflikts für Gustl wäre es demnach gewesen, den Bäckermeister durch Bedrohung mit gezogener Waffe auf der Stelle und öffentlich zum Widerruf der Beleidigung zu zwingen. ${ }^{22}$ Um es mit den Worten eines zeitgenössischen Fachmanns in Sachen Ehrenkodex zu sagen, war es die absolute Pflicht eines beleidigten Offiziers, die Beleidigung mit der Waffe zu rächen, 
"da sich dieser gemäß den im Offizierskorps herrschenden Anschauungen der unzureichenden Verteidigung seines Ehrenkleides und somit der Otfiziersehre schuldig machen würde, wenn er sich nicht der zuständigen Waffe bediente, um das Prestige seines Standes vor einem Schimpf zu bewahren und den Beleidiger zu züchtigen." 23

Einen historischen Parallelfall dazu schildert Casanova, 24 der 1750 in Vicenza Zeuge einer Auseinandersetzung zwischen einem kaiserlichen Hauptmann und einem Gastwirt wurde, bei der der Wirt dem Offizier das Gästebuch um die Ohren schlug: "Als ich sah, wie diese Memme die Beleidigung hinnahm, ohne sich zu erinnern, daß er einen Degen trug und in einer Uniform steckte, verließ ich den Raum [. . . " Casanova lehnte hinfort jede weitere Beziehung zu dem Ehrlosen ab. Einen literarischen, radikaleren und abstoßenderen Parallelfall liefert der Oberleutnant Karinski in Schnitzlers Drama Freiwild, der "einen Zivilisten im Kaffeehaus beinah zusammengehauen" hat: "Was hä̈t' ich denn tun sollen, wenn so ein Kerl im Restaurant an mich anstoßt und es nicht einmal der Mühe wert hält, Pardon zu sagen. . . es war meine Pflicht, so zu handeln. ${ }^{.25}$

Durch den körperlich überlegenen Bäckermeister wird Gustl aber an der Ausübung seines Gewaltmonopols gehindert. Da er seine soziale Überlegenheit nicht durchsetzen kann, ist die Ehrenkränkung also vollzogen und nicht revozierbar. [Notabene: wir reden von "Sie dummer Bub", nicht von "Halten Sie das Maul". Was ein Zivilist sich von einem Offizier gefallen lassen mußte, steht auf einem anderen Blatt.] Ein Duell kann nicht stattfinden, da der Bäckermeister als Handwerker nach dem Ehrenkodex der Armee nicht gleichrangig, also nicht satisfaktionsfähig ist.

Trotzdem ist Gustls selbstmitleidiges "Ganz wehrlos sind wir gegen die Zivilisten" (347) nicht gerechtfertigt, da er selbst durch seine Frechheit den Streit provoziert hat und also am Konflikt selbst schuld ist. Zwar ist ein Appell an den Ehrenrat zwecklos, aber auch an einer zivilrechtlichen Beleidigungsklage gegen den Bäcker kann Gustl keinesfalls gelegen sein, da bei dieser sowohl der Wortlaut der Beleidigung als auch sein eigenes ausfallendes Verhalten in die Öffentlichkeit dringen würden. ${ }^{26}$ Das Gewaltmonopol des Duellkodex, ursprünglich Privileg zur Absonderung der exklusiven Führungsschicht von der Masse, scheint allerdings nur in diesem speziellen Fall und unter den gegebenen Bedingungen gegen Gust zu diskriminieren, wenn man außer Acht läßt, daß Gustl den Konflikt, dem er zum Opfer zu fallen droht, ganz durch eigene Schuld heraufbeschworen hat.

Unter diesen Umständen bleiben Gustl nur die beiden Möglichkeiten offen, die er im Laufe der Nacht immer wieder durchspielt: 
a. Er kann als Entehrter den Abschied vom Militär nehmen und sich im Sinne seines früheren Status als "Unperson" ins Privatleben zurückziehen oder gar auswandern Möglichkeiten, die sich ihm von selbst verbieten, weil er nach eigener Einsicht für das praktische Leben untauglich ist und überdies nach einer ganz auf die Offiziersehre gestellten Karriere mit seiner Ehre auch seine Selbstachtung verlieren würde. So sehr hat er den Ehrenkodex seiner Kaste internalisiert. Diese Ausweglosigkeit ist im Charakter der Figur angelegt und steht als solche nicht weiter zur Debatte.

b. Er kann es vorziehen, sein Versagen, auf eine Herausfordenung mit angemessenen Ehrenmitteln zu reagieren, an sich selbst zu sühnen, ohne das Prinzip anzutasten, also in simplen Worten: seine Entehrung nicht überleben zu wollen und den Freitod zu wählen, der inm paradoxerweise, da er die Ehre über das Leben stellte, ein militärisches Ehrenbegräbnis sichern würde.

In dieser Wahl zwischen dem moralischen Tod als Ehrenmann und dem physischen Tod als Person entscheidet sich Gustl in einem Augenblick der Wahrheit und der Selbsterkenntnis für den letzteren Weg. In dieser Hinsicht hat Gustl einen Wesensverwandten in dem jüdischen Reserveoffizier Oskar Ehrenberg in Schnitzlers Der Weg ins Freie. Von seinem eigenen Vater öffentlich geohrfeigt, kann dieser weder den Vater zum Duell fordern noch die öffentliche Ohrieige ungesühnt hinnehmen. Er unternimmt daher in vollkommener Übereinstimmung mit dem Ehrenkodex einen Selbstmordversuch, der ihn ein Auge kostet, aber die Leutnantscharge rettet. 27

Es ist aber notwendig, hier nochmals deutlich festzuhalten, daß das Verhalten des Bäckermeisters nicht bezweckt hatte, Gustl in den Selbstmord zu treiben. Der Bäckermeister hat offensichtlich vielmehr in besserer Einschätzung der Person und der Sachlage berücksichtigt, daß die Vertraulichkeit der Auseinandersetzung unter Ausschluß der Öffentlichkeit Gustl den Weg zu mancherlei Kompromissen oder optimalerweise sogar tieferen Einsichten eröffnen würde, und seine Einschätzung wird durch den Ausgang der Novelle, wenn auch nur zur einen Hälfte, bestätigt.

An diesem Punkt weicht wiederum ein Teil der Interpreten von der von Schnitzler vorgezeichneten Entwicklungslinie ab, und $z$ war in dem Sinne, als sei es nur das öffentliche Ansehen, die Reputation, das Sozialprestige oder, wie das 19. Jahrhundert sagte, die "äußere Ehre", deren Kränkung den Selbstmord erfordert. Aứ die bestürzend einfachste Formel bringt es Robert Blauhut: "Gustl muß sich umbringen, wenn es ruchbar wird."28 Zwar räsonniert auch Leutnant Gustl gleich nach der Auseinandersetzung:"Wenn's ein Mensch gehört hätt', so müßt ich mich ja stante pede 
erschießen." (344) Aber das ist nur seine allererste - und freilich auch später seine letzte - Reaktion. Zwischendurch hat Gustl lichte Momente, in denen sein Wille und der Weltwille der Konvention unisono verlaufen: ". . . es ist doch ganz egal, ob ein anderer was weiß! . . I Ich weiß es doch, und das ist die Hauptsache! Ich spür', daß ich jetzt wer anderer bin, als vor einer Stunde - Ich weiß, daß ich satisfaktionsunfähig bin, und darum muß ich mich totschießen ..." (346). Und es gibt Momente, wo er aus der Notwendigkeit des Freitodes überdies melodramatisch persönliches Kapital an Ehre schlägt:

Und wenn ihn heut nacht der Schlag trifft, so weiß ich's . . . ich weiß es . . . und ich bin nicht der Mensch, der weiter den Rock trägt und den Säbel, wenn ein solcher Schimpi auf ihm sitzt! (348)

Dieses Zitat mutet wie eine ironisch umgekehrte Vorwegnahme des Endes an und ist es auch: Nie wieder sieht Gustl seine Situation in Bezug auf den Ehrenpunkt mit solcher Klarheit, aber schließlich soll der bloße Überlebenswille Bedenken dieser Art verstummen machen.

Denn die hier nur potentiell angedeutete Möglichkeit tritt gegen Ende ja tatsächlich ein. Nach einer Nacht des Umherirrens im Kreise, im Raum wie in Gedanken, und einem knappen Abstecher in eine Kirche kehrt Gustl zu seinem letzten Frühstück eine ebenso elegante Umkehrung des last supper wie Gustls nächtlicher Osterspaziergang durch die Großstadt - in seinem Stammkaffee ein und erfährt vom Kellner, den Bäckermeister Habetswallner habe "heut' nacht um zwölf der Schlag getroffen" (364). Noch eine Zusatzfrage "Ist er tot?" und die liebenswürdige Versicherung des charmanten Menschenfreundes "Na freilich, Herr Leutnant; auf'm Fleck ist er tot geblieben." (365)

Gustls Reaktion ist absehbar. Wie er früher allen anderen die Schuld an seinem Unglück gegeben hat, der Steffi, dem Kopetzky, dem Ballert, ja selbst dem Freibillet, so will er für sein Glück ganz allein verantwortlich sein: "Und das Mordsglück, daß ich in das Kaffeehaus gegangen bin" (365f.) - "O herrlich, herrlich! - Am End' ist das alles, weil ich in der Kirchen g'wesen bin." (365) Und natürlich: "Keiner weiß was, und nichts ist g'scheh'n! . . er ist tot, und ich darf leben, und alles g'hört wieder mein!" (365f.)

Was ist eigentlich geschehen? Den Beleidiger von Gustls Ehre, wenn wir ihn einmal mit Gustls Augen so sehen wollen, hat Stunden nach dem Delikt der Schlag getroffen. Glücklicherweise hat keiner der Interpreten das als ein Gottesurteil verstanden, und 
selbst Gustl würde wohl eine solche Deutung nicht aufrechterhalten können, da der Tod des Bäckers seinem Kirchgang um sechs Stunden vorausging, und soweit greift die göttliche Providenz denn doch wohl nicht voraus. Aber da Gustl sein Glück nicht dem Zufall verdanken, sondern daran teilhaben will, sieht er sich selbst als Mittel der Vorsehung, sich an seinem Beleidiger zu rächen: "Am End' hat ihn der Schlag getroffen aus Wut, aus verhaltenem Zorn. . . " (366) Immerhin eine Interpretation, die Zukunft hat: Daß Gustl sein Leben der Tatsache zu verdanken habe, daß der Bäcker sich bei dem Recontre so beherrscht habe, während der Bäcker seinen Tod dem Umstand verdanken solle, daß Gustl sich nicht beherrscht habe. Wir stellen anheim.

Eine solche Deutung würde dem Dilemma einiger Interpreten abhelfen, die einen Mangel darin sehen, daß es gerade der Deus ex machina-Charakter des Zufalls sein soll, der Gustls Leben eine neue Wendung gibt - ohne Einsicht dafür, daß es gerade ein Zufall sein muß, damit nämlich nicht der geringste ursächliche Zusammenhang zwischen der Konfliktszene und dem Tod des Bäckers entstehen darf.

Ist Gustls Ehre damit wiederhergestellt, enthebt inn der zufällige Tod des Beleidigers nunmehr seines Selbstmordes? Auch hier erweist sich der innere Monolog als eine gefährliche Erzähltechnik, insofern er falscher Einfühlung und Sympathie mit dem Sprecher Vorschub leistet, und ein Großteil der Interpreten macht entsprechend Gustls Argumentation unversehens zu der ihrigen. Nur ein paar Beispiele:

"The baker at midnight died of a stroke. Thus Gustl is saved."29

"[der] plötzliche Tod, der den Leutnant der Notwendigkeit des Selbstmordes enthebt"30

"Durch dieses 'Mordsglück' sieht sich der Leutnant von Schmach und Schande befreit - und kann weiter in den Tag hinein leben. ${ }^{31}$

"Der Deus ex machina, der den Leutnant schließlich vom Selbstmord suspendiert [. . . .]"32

"Gustl ist dem Leben wiedergegeben"33

"der [. . .] durch einen blindgnädigen Schicksalseingriff vom Tode unerwartet freigesetzte Leutnant" 34

"and hence he is relieved of the necessity to commit suicide"35

"Gereinigd van smaad en schande kan hij nu verder leven." 36

"Am Morgen wird Gustl wie durch ein Wunder gerettet [. . . ] Da der Beleidiger tơ ist, kann der Beleidigte weiterleben." 37

"[Gustl muß] den Vorsatz, sich umzubringen, nicht durchführen"38 
[Der Tod des Bäckers] "erlaubt ihm, weiterzuleben"39

Selbst Gero von Wilpert ${ }^{40}$ schreibt noch 1968 - und das ist natürlich gar nicht mehr originell - daß "ein bloßer Zufall den Tod unnötig macht". Und bei Renate Wagner schließlich wird Gustl sogar jene anfangs apostrophierte Unsterblichkeit zugesichert, wenn sie meint, daß "der Zufall, der Tod des Bäckermeisters, ihn des eigenen Todes enthebt." 41

Angesichts einer so auffallenden Übereinstimmung der Interpreten bis in den Wortlaut hinein könnten einen Bedenken beschleichen, die Dinge vom Kopf auf die Füße zu stellen.

Rezeptionsgeschichtlich gesehen, stelt sich dabei die Frage, ob Schnitzlers Novelle noch soweit aktuell ist, daß wir sie, wie viele Interpreten es tun, mit unserer modernen Werteskala messen können, oder ob sie schon soweit historisch zeitgebunden ist, daß ihr volles Verständnis erfordert, sich in die Werteskala der höheren Gesellschaft um die Jahrhundertwende zurückzuversetzen. Im letzteren Fall bliebe den Interpreten der Vorwurf nicht erspart, daß sie den Charakter des inneren Monologs zeitlos verabsolutiert haben und sein Präsens für ihre Gegenwart, sein Ich und seine Meinungen für ihr Ich und ihre Meinungen genommen haben.

Tatsache jedenfalls ist, daß der Tod des Bäckers als Zufall in keinem nachweislichen Zusammenhang mit dem Konflikt steht. Tatsache ist ferner, daß Gustls Ehre weder in der Zwischenzeit noch durch den Tod des Bäckers wiederhergestellt worden ist.

Ist denn ein Ehebruch nicht verübt worden, wenn den Ehebrecher tags darauf der Schlag trifft, hat ein Einbruch nicht stattgefunden, wenn der Einbrecher auf der Flucht einem Verkehrsunfall zum Opfer fällt, ist ein Mord nicht geschehen, wenn den Mörder ein Blitz erschlägt? Einmal angenommen, Gustl hätte sich nicht umgebracht und der Bäcker wäre erst zwei Wochen später verstorben, hätte Gustl dann zwei Wochen lang ehrlos gelebt und wäre dann rehabilitiert worden?

Die Beispielreihe praktischer Lebensweisheit erübrigt sich. Denn Gustl, im Moralund Ehrenkodex seiner Zeit genauer bewandert als seine heutigen Leser, hat ja selbst schon einen lichten Moment lang die richtige Einsicht gehabt, daß auch der Tod des Bäckers an seiner Entehrung nichts ändern würde. (348)

Dies ist kein Plädoyer für eine enthumanisierte Literaturwissenschaft. Wir wollen nicht blutrünstig auf dem Selbstmord Gustls bestehen, sondern ihm sein Weiterleben gönnen. Nur: Seine Ehre hat er nach dem Gesetz, nach dem er angetreten, verloren, und sie ist auch durch das zufällige Ableben des Kontrahenten nicht wieder hergestellt worden. Wenn wir uns auch nur historisch in den Komment und die Ehrpusseligkeit der 
Jahrhundertwende zurückversetzen können: Gustl war sich dessen bewußt, oder es wird inm bestenfalls später einmal wieder oder, wahrscheinlicher noch, aus guten Gründen nie bewußt werden, daß der Tod des Bäckers an seiner verletzten Ehre nicht das geringste ändert.

Für Gustl gibt es eine Entschuldigung, die keine Rechtfertigung ist: sein Überlebenswille, den man ihm so oft abgesprochen hat, ist stärker als die Vorschrift der Konvention. Und das ist an sich nichts Schlechtes, wenn er die Kraft hätte, sich wenigstens insgeheim darüber im klaren zu sein, daß er um des Überlebens willen die Konvention geopfert hat, daß er sich gegen die Konvention gewandt und andere Werte ihr vorgezogen hat, daß er seinen einzigen moralischen Rückhalt, den Ehrenkodex, geopfert hat zugunsten eines Lebens, dem allein dieser Ehrenkodex noch Glanz und Sinn verleihen konnte. Das geschieht jedoch nicht.

Leutnant Gustl nimmt nicht wie Lessings Major von Tellheim seine Existenz solange aus der Öffentlichkeit zurück, bis seiner Ehre volle Genugtuung geschehen ist. Welten trennen den sensitiven adligen baltischen Major, dessen Vornamen wir nicht kennen, von dem kleinbürgerlichen österreichischen Leutnant, den wir nur beim Vornamen kennen. Der eine beantwortet den Zweifel an seiner Ehre mit der Zurücknahme seines sozialen lchs, der andere mit einer Duellforderung. Der eine macht aus seiner Ehrenkränkung keinen $\mathrm{Hehl}$, der andere verdrängt und vertuscht sie. Die Unterschiede werden noch deutlicher, wenn der eine ohne Ehre nicht das Leben einer liebenden und geliebten Frau an sich zu binden wagt, während der andere, auch ohne Ehre, das Leben eines Außenstehenden zu vernichten ansetzt. Aus der Ehre, die den Kern des Menschen bestimmte, ist ein bloßer veräußerlichter Popanz geworden, der den inneren Menschen so wenig berührt, daß er gut ohne ihn auskommt, solange es nur keiner weiß.

So also, in Schnitzlers Novelle, gilt Gustl nur deshalb noch als Ehrenmann, weil die Beleidigung seiner Ehre keine Zeugen hatte; weil es keiner weiß und weil seine späteren Interpreten es ihm bei gewandelter Ehrauffassung nachsehen wollen. "Wenn's einer sieht, so paßt er gerade so wenig auf, wie ich." (337) So begann die Novelle.

Wozu hat sie geführt? Ist Gustl durch die Konfrontation mit dem Tod zu tieferer Einsicht gelangt wie Hofmannsthals Tor, hat er sich in der Todesbegegnung gewandelt und geläutert, hat er seinen Erfahrungshorizont enweitert, ist er ein besserer Mensch geworden? Viele Interpreten machen inm die fehlende Wandlung zum moralischen Vorwurf und sehen darin überhaupt die Aussage der Novelle, wobei wiederum kaum berücksichtigt wird, daß Gustl ja nicht das erstemal dem Tode gegenübersteht.

Und wenn man zwischen Duelltod und Selbstmord differenzieren will, ist ihm dann 
wenigstens die Fragwürdigkeit und Scheinhaftigkeit seiner Ehren-Ideologie zu Bewußtsein gekommen, hat er die Konventionen, die er hintergeht, auch hinterfragt?

Der Ablauf des inneren Monologs macht eines erschreckend deutlich, nämlich nicht nur den inneren Leerlauf dieser Existenz, sondern auch die Unfähigkeit, aus ihr auszubrechen und aus dem Einerlei von Spielschulden, Ehrenhändeln und Frauenaffären zum eigentlichen Leben durchzustoßen. Viele Interpreten bemühen zur Verdeutlichung dieser Situation die Struktur des Reigens: Gustl bewege sich, zur Entscheidung aufgerufen aber unfähig zur Antwort, nur im Kreise, ${ }^{42}$ er umgehe bewußt oder unbewußt die ehrliche Auseinandersetzung und sei am Ende derselbe wie am Anfang. ${ }^{43}$

Auch diese weitverbreitete Auffassung ist mit dem vorliegenden Text nicht vereinbar und daher zumindest nur bedingt richtig, wenn nicht effektiv falsch.

In dem Wechsel von Rückblicken in die Vergangenheit und von Hoffnungen auf die Zukunft taucht ein Motiv immer wieder auf, das mit beiden Zeitebenen und darüberhinaus auch mit dem Begriff der Ehre aufs engste verbunden ist, nämlich das bevorstehende Duell mit dem Doktor.

Zu Beginn der Novelle will Gustl ihm nur eine Lektion erteilen, ohne größere Ansprüche an seine Körperlichkeit zu stellen: "Warten S' nur, Herr Doktor, Ihnen wird's vergeh'n, solche Bemerkungen zu machen! Das Nasenspitzel hau' ich Ihnen herunter." (338)

Solche Zielsicherheit hält freilich nicht lange vor. Noch während des Oratoriums steigen bei abnehmendem Zorn mit den Frustrationen auch die Ansprüche an den Duellausgang: "Wart' nur, mein Lieber - bis zur Kampfunfähigkeit . . . jawohl, du sollst so kampfunfähig werden. .." (341) Löbliche Vorsätze, die Bezweifler militärischer Ehre mehr als nur mundtot zu machen!

Nach der Konfrontation mit dem Bäcker ist Gustl sich bewußt, satisfaktionsunfähig zu sein (346), und befürchtet, die Sekundanten könnten ihm ihr Mandat zurückgeben (347). Nach dem Tod des Bäckermeisters und einer Nacht der verpaßten Gelegenheiten zu erbaulicher Selbsterforschung aber liest man's anders: "Wart', mein Lieber! Ich bin grad' gut aufgelegt ... . Dich hau ich zu Krenfleisch!" (366)

Ich bin nicht der Meinung, daß dies ein Kreislauf ist, der in die Anfangsphase mündet. Die Bedrohung des eigenen Lebens und die Einsicht in die Richtigkeit der Aussage des Doktors haben Gustl nicht zu abgeklärter Toleranz und Einsicht in menschliche Schwächen geführt. Vielmehr führt der heimliche geistige Offenba. rungseid in dem Augenblick, wo die Fassade wieder intakt ist, zu erhöhtem Aggres- 
sionsstau und gesteigerter Brutalität der Kampfeslust.

Doch jetzt geht es nicht mehr um einen Ehrenhandel zwischen zwei satisfaktionsfähigen Kontrahenten. Der Doktor, der laut Gustl die Ehre der Armee verletzt hat, wird am kommenden Nachmittag, ohne es zu wissen, einem nicht satisfaktionsfähigen Gegner gegenüberstehen. Es kann gar nicht davon die Rede sein, wie Politzer ${ }^{44}$ meint, daß Gustl sich zu dem Duell mit dem Doktor "jetzt wieder stellen darf". Gustl ist und bleibt nach militärischem Komment entehrt und damit nicht satisfaktionsfähig, und an dieser Sachlage ändert sich dadurch nicht das Geringste, da $\beta$ niemand etwas davon weiß. Der für Gustl günstige Duellausgang steht, wenigstens in Gustls Bewußtsein, unbezweifelbar fest, und durch die prononzierte Endstellung des "Krenfleisch"-Vorsatzes soll auch dem Leser jeder Zweifel am Ausgang genommen werden.

Was Gustl als Ehrenhandel eigentlich nur aufgegriffen hatte, um bei seinen Vorgesetzten Ehre einzulegen und Pluspunkte zu sammeln - denn seine persönliche Ehre war ja gar nicht angegriffen worden - wird jetzt, da der ehrlose Gustl seine Verzweiflung, seine Frustration und seine Aggressionen an einem unschuldigen Opfer abzureagieren ansetzt, zu einer Racheaktion am falschen Objekt, ja zu einem vorsätzlichen und brutalen Mord eines Ehrenmannes durch einen Ehrlosen. Je weniger Anlaß besteht, auf seine eigene Ehre zu pochen, umso radikaler und brutaler werden die Maßnahmen, die nach außen hin der trügerischen Aufrechterhaltung des gar nicht mehr Vohandenen dienen.

Das ist das wahre Gesicht von Gustls Ehre.

Angesichts der vielen unfundierten und irreführenden Deutungen von Gustls Ehre ist man schließlich versucht, der Motivstudie des Ehrbegriffs noch den philologischen Segen einer Untersuchung des Wortgebrauchs nachzuliefern. Das führt zu erstaunlichen Einsichten, die die stilistische Kunst von Schnitzlers sprachlicher Orchestration bezeugen.

Es wäre ja anzunehmen, daß in einem Text, der so stark die Ehre thematisiert, das Wort "Ehre" und seine Zusammensetzungen einen überdurchschnittlichen Rang einnähmen. In Brentanos etwa gleichlanger Geschichte vom braven Kasperl und dem schönen Annerl, in der es ja ähnlich um falsche und wahre Ehre geht, kommen das Wort "Ehre" und Komposita allein siebenundsiebzigmal vor, das heißt mehr als zweimal je Seite. In Schnitzlers Novelle dagegen erscheint es nicht mehr und nicht weniger als elfmal, davon dreimal in Komposita (Ehrenwort, Ehrenrat) und zweimal 
in sprichwörtlichen Wendungen (Ehre verloren, alles verloren; das Feld der Ehre), selbständig dagegen nur sechsmal, und zwar immer nur in ein und derselben Wendung, die der Ironie des Ganzen die Krone aufsetzt. Die Wendung heißt: "Habe die Ehre!" $(342,344,361,364)$

Gerade diese bestürzende Feststellung, daß das Wort "Ehre" in Gustls Sprach- und Begriffsschatz gar nicht mehr vorkommt außer in belanglosen, banalen Floskeln, die ebenso ausgehöhlt sind wie der Ehrbegriff, gibt zu bedenken. Wenn man auf die andere Banalität zurückgreift, daß Begriffe wie Kultur und Ehre nur von solchen Leuten ständig im Munde geführt werden, die sie nicht haben, dann ist Gustl schon einen Schritt weiter: er redet nicht einmal mehr davon.

Schnitzlers Kritik am hohlen, oberflächlichen Ehrenkodex seiner Zeit streitet nicht die Existenz eines idealen Ehrbegriffs ab, sondern nur seine Relevanz für die zeitgenössische Gesellschaft, die sich in veräußerlichten Konventionen und Verhaltensregeln Genüge tut, ohne deren Grundbegriffe wie Ehre zu hinterfragen. Sonst wäre selbst Gustl sicher darauf gekommen, daß im Verhalten des Bäckermeisters mehr Ehre und Würde lag als in seinem schrillen Schneid.

Wenn nun aber die Exerziergriffe der "Ehre" eingepaukt werden, ohne daß ihr Wesen befragt und hinterfragt wird, wenn es nur noch, mit Fontanes Baron von Innstetten zu reden, das Gesellschafts-Etwas ist, das die Konventionen aufrechterhält, dann ist das letzte Stadium solcher Veräußerlichung der Gesellschaft erreicht. Der Verfall beginnt in dem Augenblick, wo die Konvention weiterbesteht, der Begriff Ehre aber pervertiert oder abhandengekommen ist, und das ist bei Leutnant Gustl der Fall.

Man könnte hier aufhören und wäre glücklich, könnte man es. Aber die Geschichte hat wie jede gute Geschichte noch ein Nachspiel. Schnitzler, der selbst als Arzt den Rang eines Reserveoffiziers der k. u. k. Armee bekleidete, wurde drei Tage nach der Veröffentlichung des Leutnant Gustl in dem Offizierskreisen nahestehenden, konservativen Blatt Die Reichswehr durch dessen Chefredakteur Gustav Davis heftig persönlich attackiert. Er reagierte darauf nicht, wie von einem Reserveoffizier zu erwarten war, mit einer Duellforderung, weil er zeitlebens Gegner des Duells und des Duellzwangs war. Auch einer dreifachen Vorladung vor einen militärischen Ehrenrat leistete er keine Folge, weil er einem Militärgericht kein Urteil über seine literarischen Werke zubilligte. Daraufhin wurde er am 21. Juni 1901 wegen Verletzung der Standesehre seines "Offizierscharakters für verlustig erklärt". 45

Der Tragödie ist damit das Satyrspiel gefolgt. Gustl hat zurückgeschlagen und 
gewonnen. Er behält, vor den Augen der Welt wenigstens, seine Offiziersehre und wird ihr im gleichen Sinne wie bisher Ehre machen. Der Doktor, der zur Ehrlichkeit mahnte, ist zu Krenfleisch verarbeitet, der Autor, der für Wahrhaftigkeit in Ehrensachen plädierte, seiner Offiziersehre entkleidet. Und man vermag abschließend nur die Weisheit eines militärischen Ehrenrats zu bewundern, der erkannte, daß ein Ex-Offizier, der mehr ist als bloß Offizier, auch ohne seine Offiziersehre ein angesehener Mann sein kann, und der der Nachwelt den Autor Arthur Schnitzler erhielt und ihr einen Arthur Krenfleisch ersparte.

Anmerkungen

1 Sekundärliteratur:

Otto P. Schinnerer, "Schnitzler and the Military Censorship. Unpublished Correspondence.", GR 5,3 (1930), S. 238-246.

Richard H. Lawson: "A Reinterpretation of Schnitzler's Leutnant Gustl', Journal of the International Arthur Schnitzler Research Association 1,2 (1962), S. 4-19.

Hellmuth Himmel, Geschichte der deutschen Novelle, Bern 1963, S. $364 f$.

Wilfried F. Schoeller, "Lieutenant Gustl", Kindlers Literatur Lexikon, Band IV, Zürich 1964, Spp. 1407-1409.

Manfred Jäger, "Schnitzlers Leutnant Gust", WW 15 (1965), S. 308-316.

Arthur Schnitzler, Liebelei, Leutnant Gustl, Die letzten Masken, hrsg. von Joseph Peter Stern, Cambridge 1966. Introduction S. 28-40.

Françoise Derré, L'oeuvre d' Arthur Schnitzler. Imagerie viennoise et les problèmes humains, Paris 1966, S. 155-159.

Robert Blauhut, Österreichische Novellistik des 20. Jahrhunderts, Wien 1966.

Heinz Politzer, "Diagnose und Dichtung. Zum Werk Arthur Schnitzlers", H. P., Das Schweigen der Sirenen, Stuttgart 1968, S. 110-141 (zuerst in Arthur Schnitzler, Leutnant Gustl, hrsg. von H.P., Frankfurt 1962, S. 40-50).

Gero von Wilpert, "Leutnant Gustl", Lexikon der Weltliteratur, Band II: Werke, hrsg. ders. Stuttgart 1968, S. 642.

Theodore W. und Beatrice W. Alexander, "Schnitzler's Leutnant Gustl and Dujardin's Les lauriers sont coupés", MAL 2, 2 (1969), S. 7-15.

Hans Ulrich Lindken, Interpretationen zu Arthur Schnitzler. Drei Erzählungen, München 1970, S. 76-99.

Martin Swales, Arthur Schnitzler. A Critical Study, Oxford 1971, S. 103-114. Alfred Doppler, "Innerer Monolog und soziale Wirklichkeit. Arthur Schnitzlers Novelle Leutnant Gustr", A. D., Wirklichkeit im Spiegel der Sprache, Wien 1975, S. 53-64 (zuerst in: Interpretationen zur österreichischen Literatur. Wien 1971, S. 53-61).

J. P. J. Maassen, "Lieutenant Gustl", Moderne Enzyclopedie der Wereldliteratuur, Band VII. Gent 1972, S. 576

Hartmut Scheible, Arthur Schnitzler, Reinbek 1976. 
Friedrich Knilli, "Lieutenant Gustl - ein k. u. k. Antisemit aus bundesrepublikanischer Sicht", Literatur in den Massenmedien, hrsg. ders. München 1976, S. 139-164.

Rolf-PeterJanz und Klaus Laermann, Arthur Schnitzler. Zur Diagnose des Wiener Bürgertums im Fin de siècle, Stuttgart 1977, S. 110-131.

Josef Kunz, Die deutsche Novelle im 20. Jahrhundert, Berlin 1977, S. 52-61.

Nils Ekfelt, "Schnitzler's Leutnant Gustl: Interior monologue or interior dialogue?", Sprachkunst 11 (1980), S. 19-25.

Dirk Dethlefsen, "Überlebenswille: Zu Schnitzlers Monolognovelle Leutnant Gustl in ihrem literarischen Umkreis", Seminar 17 (1981), S. 50-72.

Rolf Geißler, "Bürgerliche Literatur am Ende. Epochalisierung am Beispiel von drei Erzählungen Schnitzlers", R. G. , Arbeit am literarischen Kanon. Paderborn 1982, S. 115-137.

Renate Wagner, Arthur Schnitzler. Eine Biographie, Frankfurt 1984.

Roland Duhamel, "Arthur Schnitzlers Modernität - am Beispiel von Leutnant Gust", Germanistische Mitteilungen 19 (1984), S. 19-24.

Alle Zitatbelege im Text nach Arthur Schnitzler, Die erzählenden Schriften, Band I, Frankfurt 1961.

2 Lindken, S. $91 f$.

3 Laermann, S. 117, Knilli, S. 148.

4 Kunz, S. $54 f$.

5 Lawson, S. 14.

6 Lawson, S. 16.

7 Lawson, S. 17.

8 Lawson, S. 8, Laermann, S. 119.

9 Lawson, S. 6, Politzer, S. 123.

10 Laermann, S. 121.

11 Lawson, S. 7, S. 9.

12 Lawson, S. 11.

13 vgl. Knilli, S. $151 f$.

14 Arthur Schnitzler, Die dramatischen Werke, Band I, Frankfurt 1962, S. 90.

15 "Brief an Theodor von Sosnosky", Neues Wiener Journal vom 26.10.1931, zitiert nach Dethlefsen, S. 50.

16 Dichtung und Dichter der Zeit, 19. Aufl. 1928, S. 508. 
17 Adalbert Schmidt, Dichtung und Dichter Österreichs im 19. und 20. Jahrhundert, Salzburg und Stuttgart 1964, Band I, S. 243.

18 dazu ausführlich Laermann, S. 114.

19 Kunz, S. 57.

20 Albert Soergel und Curt Hohoff, Dichtung und Dichter der Zeit, Band I, 1961, S. 446.

21 Lindken, S. 87.

22 dazu Laermann, S. 131.

23 Josef Edler von Seidel, Der Zweikampf . .., Laibach 1896, zitiert nach Laermann, S. 143.

24 Geschichte meines Lebens, Berlin 1965, Band III, S. 340.

25 Arthur Schnitzler, Die dramatischen Werke, Band I, Frankfurt 1962, S. 273, S. 287.

26 ebenso Laermann, S. 131.

27 Arthur Schnitzler, Die erzählenden Schriften, Band I, Frankfurt 1961, S. 808, S. $814 f$., S. 822 , S. 825 , S. $843 f$.

28 Blauhut, S. 23.

29 Lawson, S. 5.

30 Himmel, S. 364.

31 Schoeller, Sp. 1408.

32 Jäger, S. 308.

33 Blauhut, S.23.

34 Lindken, S. 94.

35 Swales, S. 103.

36 Maassen, S. 576.

37 Doppler, S. $55 f$.

38 Scheible, S. 81.

39 Laermann, S. 113. 
40 von Wilpert, S. 642.

41 Wagner, S. 114.

42 z. B. Jäger, S. 308, S. 315, Lindken, S. $80 f .$, Duhamel, S. 23.

43 z. B. Politzer, S. 127, Lindken, S. 80 , S. 94 , Doppler, S. 56 , S. 6 of.

44 Politzer, S. 127.

45 vgl. die Einzelheiten bei Schinnerer und Wagner, S. 121-128. 